\title{
Long-Term Optical and Spectral Variability of FSRQ 3C454.3
}

\author{
Yu-Hai Yuan $\mathbb{D}^{1,2}$ Ze-Xuan Chen $\mathbb{D}^{1},{ }^{1}$ and Yong-Xuan He $\mathbb{D}^{1}$ \\ ${ }^{1}$ Center for Astrophysics, Guangzhou University, Guangzhou, Guangdong 510006, China \\ ${ }^{2}$ Astronomy Science and Technology Research Laboratory of Department of Education of Guangdong Province, \\ Guangzhou 510006, China \\ Correspondence should be addressed to Yu-Hai Yuan; yh_yuan@gzhu.edu.cn
}

Received 23 June 2018; Revised 17 September 2018; Accepted 31 October 2018; Published 2 December 2018

Academic Editor: Geza Kovacs

Copyright (C) 2018 Yu-Hai Yuan et al. This is an open access article distributed under the Creative Commons Attribution License, which permits unrestricted use, distribution, and reproduction in any medium, provided the original work is properly cited.

3C454.3 is a widely studied and monitored flat spectrum radio quasar. We use the observations from the Small and Moderate Aperture Research Telescope System (SMARTS) to analyze the long-term optical and spectral variabilities of 3C454.3 at B, V, R, J, and $\mathrm{K}$ (BVRJK) bands. Based on the relation: $F_{\gamma} \propto v^{-\alpha}$, we calculate the multiband spectral indices $(\alpha)$ and analyze the relations between $\alpha$ and $F_{v}$, where $F_{v}$ is the flux density at $\nu$ band ( $\left.\nu=\mathrm{B}, \mathrm{V}, \mathrm{R}, \mathrm{J}, \mathrm{K}\right)$. Main results are as follows. (1) The largest variations at BVRJK bands are $\Delta \mathbf{B}=\mathbf{3 . 3 7} \pm \mathbf{0 . 0 8} \mathbf{m a g}$, with the timescale $\Delta T_{B} \approx 1015$ days, at $\mathbf{B}$ band; $\Delta \mathbf{V}=\mathbf{3 . 3 1} \pm \mathbf{0 . 0 7} \mathbf{m a g}$, with the timescale $\Delta T_{V} \approx 1014$ days, at $\mathbf{V}$ band; $\Delta \mathbf{R}=\mathbf{3 . 6 2} \pm \mathbf{0 . 1 2} \mathbf{~ m a g}$, with the timescale $\Delta T_{R} \approx 679$ days, at $\mathbf{R}$ band; $\Delta \mathbf{J}=\mathbf{4 . 0 8} \pm \mathbf{0 . 0 1} \mathbf{~ m a g}$, with the timescale $\Delta T_{I} \approx 763$ days, at $\mathbf{J}$ band; and $\Delta \mathbf{K}=\mathbf{5 . 0 3} \pm \mathbf{0 . 0 3} \mathbf{~ m a g}$, with the timescale $\Delta T_{K} \approx 2715$ days, at $\mathbf{K}$ band. (2) We analyze the long-term BVRJK lightcurves and obtain the quasiperiodicities: $P_{1}=86.92 \pm 2.21$ days, $P_{2}=204 \pm 8.1$ days, and $P_{3}=1.24 \pm 0.19$ years. (3) Multiband lightcurves show time delays: $\tau_{V B}=0.15 \pm 0.12$ days, $\tau_{R B}=0.37 \pm 0.28$ days, $\tau_{J B}=0.58 \pm 0.40$ days, and $\tau_{K B}=$ $1.02 \pm 0.47$ days. (4) The relations between $\alpha$ and $F_{v}$ show strong correlations, which are typical RWB behaviors; when the source turns to be brighter, the spectral indices turn to be redder.

\section{Introduction}

Blazars show some extreme properties, such as violently optical variability, core dominance, and superluminal motion $[1,2]$. Blazars can be divided into two subclasses: BL Lacs and FSRQs (flat spectrum radio quasars). BL Lacs are characterized by featureless optical spectra or weak emission line [3], and FSRQs are composed of the flat-spectrum radio spectrum and show typically broad emission lines [1].

Blazars have relativistic jets. Their jets can produce the nonthermal emission, which can dominate the total observed emission. Many works analyzed the relations between spectral indices (or spectrum) and flux densities (or brightness) (e.g., [4-10]). Those studies can explain the reasons behind the characteristic of blazar variability and can also help us to constrain the emitting region. BL Lacs and FSRQs show different correlations. Generally, BL Lacs show that when the sources become brighter, the spectrums become harder and when the sources become fainter, the spectrums become softer. FSRQs show that when the sources become brighter, the spectrums become softer (redder). The redderwhen-brighter (RWB) behavior of FSRQs can be explained as the contribution from less variable, bluer accretion disk to the variable, redder jet emission.

Kirk and Mastichiadis (1999) [11] put forward that, for blazars, the spectral shape can be influenced by the synchrotron particle acceleration and synchrotron cooling. The spectral shape can reflect the intrinsic variability of jet [12-15]. Fiorucci, Ciprini, and Tosti (2004) [16] pointed out that the optical spectrum of QSOs (subclass of Active Galactic Nuclei) consisted of two components, the first one was variable $\left(\alpha_{v}\right.$, with a flatter slope), which came from synchrotron emission, and the other part was stable $\left(\alpha_{s}\right)$, which might come from the thermal emission. Fan et al. (2014) [17] pointed out that the correlation between brightness and spectral index can be influenced by the brightness intensity.

For blazars, the optical variable timescales can divide into three types: (1) the intraday variability (IDV), with the 


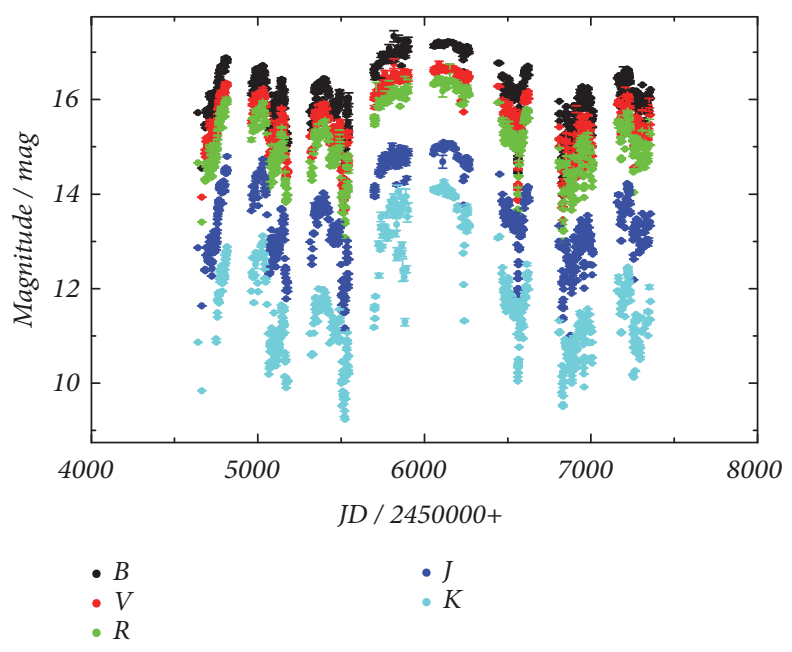

FIGURE 1: The BVRJK lightcurves of 3C454.3. The black dots stand for B band, the red dots stand for V band, the green dots stand for R band, the blue dots stand for J band, and the cyan dots stand for K band.

timescales from minutes to hours; (2) short-term variability, with the timescales of days; and (3) long-term variability, which are generally periodic, with the timescales from months to years [18]. The variable timescales can help us to study the physical processes of blazars. The IDV timescales can constrain the size of the emitting region. The long-term periodicity can give us the information about the central black hole mass of the blazars. For example, based on a slim disk, the periodicity can be determined as $P / y r=9.0 \delta(\alpha / 0.1)^{-0.62}\left(M_{B H} / 10^{6} M_{\odot}\right)$ [18], where $\alpha$ is the viscosity coefficient and $\mathrm{P}$ is the periodicity, in unit of years.

FSRQ 3C454.3 ( $\mathrm{z}=0.859$, [19]) displays strong variabilities over the entire electromagnetic bands (e.g., [20-25]). Based on the B-band lightcurve, Webb et al. (1988) [26] obtained three periodicities, $0.8,3.0$, and 6.4 years. Based on the University of Michigan Radio Astronomy Observatory (UMRAO) database, Ciaramella et al. (2004) [27] obtained a periodicity of $6.3 \pm 0.2$ years and Fan et al. (2007) [28] obtained two periodicities, $4.6 \pm 0.1$ years and $6.3 \pm 0.2$ years. Based on UMRAO and MRO (Metsähovi Radio Observatory database), Kudryavtseva and Pyatunina (2006) [29] obtained two periodicities, $P_{1}=6.2 \pm 0.1$ years and $P_{2}=12.4 \pm 0.6$ years. Woo et al. (2002) [30] availed the optical luminosity to calculate the central black hole mass being $1.47 \times 10^{9} \mathrm{M}_{\odot}$, where $M_{\odot}$ is the solar mass.

This paper is arranged as follows: in Section 2, we introduce observations and data reductions; in Section 3, results are proposed; Section 4 includes discussion and conclusion.

\section{Observations and Data Reductions}

The data used in this work are collected from the Small and Moderate Aperture Research Telescope System (SMARTS), which is located at the Cerro Tololo Inter-American Observatory (CTIO) in Chile. The data reduction and processing had been described by Brown et al. (1989) [31] and Bonning et al. (2012) [32].

For 3C454.3, the monitoring time is in the range from June 23, 2008 to November 30, 2015, and the observations cover five optical and near-IR bands, B, V, R, J, and K bands. The BVRJK lightcurves have been shown in Figure 1, which are built by

(1) 753 observations at $\mathrm{B}$ band, within $\Delta T_{B} \approx 1015$ days, the largest variations $\Delta \mathrm{B}=3.37 \pm 0.08 \mathrm{mag}$ (from $17.34 \pm 0.12 \mathrm{mag}$ to $13.96 \pm 0.002 \mathrm{mag})$;

(2) 739 observations at $\mathrm{V}$ band, within $\Delta T_{V} \approx 1014$ days, the largest variations $\Delta \mathrm{V}=3.31 \pm 0.07 \mathrm{mag}$ (from $16.76 \pm 0.10 \mathrm{mag}$ to $13.45 \pm 0.002 \mathrm{mag}$ );

(3) 745 observations at $\mathrm{R}$ band, within $\Delta T_{R} \approx 679$ days, the largest variations $\Delta \mathrm{R}=3.62 \pm 0.12 \mathrm{mag}$ (from $16.59 \pm 0.17 \mathrm{mag}$ to $12.97 \pm 0.002 \mathrm{mag})$;

(4) 744 observations at J band, within $\Delta T_{J} \approx 763$ days, the largest variations $\Delta \mathrm{J}=4.08 \pm 0.01 \mathrm{mag}$ (from $15.09 \pm$ $0.019 \mathrm{mag}$ to $11.01 \pm 0.004 \mathrm{mag}$ );

(5) 680 observations at $\mathrm{K}$ band, within $\Delta T_{K} \approx 2715$ days, the largest variations $\Delta \mathrm{K}=5.03 \pm 0.03 \mathrm{mag}$ (from $14.26 \pm 0.03 \mathrm{mag}$ to $9.24 \pm 0.002 \mathrm{mag})$.

\section{Results}

3.1. Periodicity Analysis. It is very important to choose suitable methods to analyze the long-term optical variability. Considering the uneven lightcurves, we avail the power spectrum to deal with this question and choose the common part as the quasiperiodicity. 


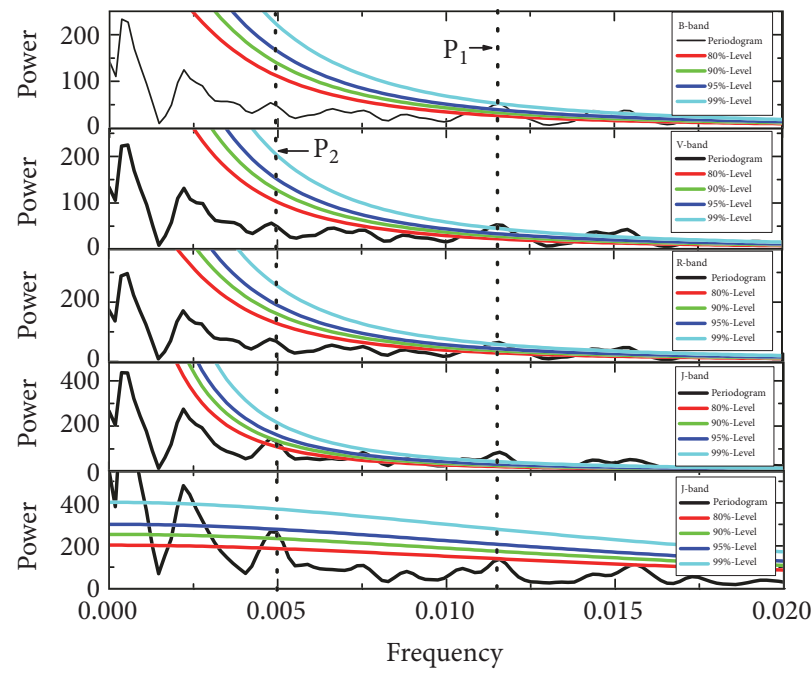

FIGURE 2: Using the Periodogram method to analyze the long-term BVRJK lightcurve. The frequency is in unit of $2 \pi \times d a y^{-1}$. The black line stands for periodic signal. The red line, green line, blue line, and cyan line stand for $80 \%, 90 \%, 95 \%$, and $99 \%$ red-noise levels, respectively.

The mostly common tool for periodicity analysis of both evenly and unevenly sampled signals is the Periodogram method, which is an estimator of the signal energy in the frequency domain by Deeming (1975) [33]. Lomb (1976) [34] introduced a modified form of this method, and additionally it was elaborated by Scargle (1982) [35], which can be described in the following. Considering a series $x(n)$ with $\mathrm{N}$ points, $\boldsymbol{f}$ is the frequency and $\tau$ is a variable timescale. Their mean and deviation are given by $\bar{x}=(1 / N) \sum_{n=1}^{N} x(n)$ and $\sigma^{2}=(1 / N) \sum_{n=1}^{N}(x(n)-\bar{x})^{2}$. The normalized Lomb's $P^{L}$, i.e., the power spectrum as a function of the angular frequency $\omega \equiv 2 \pi f>0$ is defined as

$$
\begin{aligned}
P_{N}^{L}(\omega)= & \frac{1}{2 \sigma^{2}}\left[\frac{\left[\sum_{n=0}^{N-1}(x(n)-\bar{x}) \cos \omega\left(t_{n}-\tau\right)\right]^{2}}{\sum_{n=0}^{N-1} \cos ^{2} \omega\left(t_{n}-\tau\right)}\right] \\
& +\frac{1}{2 \sigma^{2}}\left[\frac{\left[\sum_{n=0}^{N-1}(x(n)-\bar{x}) \sin \omega\left(t_{n}-\tau\right)\right]^{2}}{\sum_{n=0}^{N-1} \sin ^{2} \omega\left(t_{n}-\tau\right)}\right]
\end{aligned}
$$

and $\tau$ is defined by the equation:

$$
\tan (2 \omega \tau)=\frac{\sum_{n=0}^{N-1} \sin 2 \omega t_{n}}{\sum_{n=0}^{N-1} \cos 2 \omega t_{n}} .
$$

The errors of the period are estimated by the half width at half-maximum (HWHM) of the minimum standard deviation $\left(V_{m}^{2}\right)$. We use the Periodogram method to analyze the BVRJK lightcurves and obtain the periodicities $P_{1}=86.92 \pm$ 2.21 days, $P_{2}=204 \pm 8.1$ days, $P_{3}=1.24 \pm 0.19$ years. The periodic signals are shown in Figure 2. Red noise is a random signal, which has been filtered in order to generate a lot of energy at low frequencies. In order to check the strength of the periodic signal, we compare the periodic signal with the red noise. The red noise [36] with the noise levels $80 \%, 90 \%, 95 \%$, and 99\% is shown in Figure 2. Based on the results, we can find that at the whole five bands, $P_{1}$ is higher than $99 \%$ noise level, at J and $\mathrm{K}$ bands, $P_{2}$ is higher than $90 \%$ noise level, and only $\mathrm{K}$ band $P_{3}$ are higher than $99 \%$.

3.2. The Spectral Indices. We use the following method to obtain the spectral indices. Firstly, we make Galactic Extinction correction, using $A_{B}=0.382 \mathrm{mag}, A_{V}=0.289 \mathrm{mag}$, $A_{R}=0.228 \mathrm{mag}, A_{J}=0.075 \mathrm{mag}$, and $A_{K}=0.032 \mathrm{mag}$, which are collected from NED (http://ned.ipac.caltech.edu/); then we convert the magnitudes $\left(m_{\gamma}\right)$ into flux densities $\left(F_{\nu}\right)$, where $v$ is frequency; and at last, based on the relation $F_{v} \propto$ $\nu^{-\alpha}$, the spectral indices $(\alpha)$ can be calculated by the linear relation: $\log \left(F_{\nu}\right)=-\alpha \log (\nu)+$ const.

After calculation, there are 752 spectral indices $(\alpha)$, which are in the range from $0.33 \pm 0.01$ to $2.02 \pm 0.07$, with the averaged value $\bar{\alpha}=1.26 \pm 0.31$.

3.3. Relations between Flux Densities and Spectral Indices. Because the linear relations can clearly show the interdependence between the two parameters, so many works used the linear correlations to analyze the relations between spectral indices $(\alpha)$ and flux densities $\left(F_{\nu}\right)$. To compare with the others, we use linear correlations to analyze $\alpha=$ $\mathrm{k} \log F_{v}+b$, with the correlation coefficient $\mathrm{r}$ and the chance probability $\mathrm{p}$, the slope $\mathrm{k}$, and the intercept $\mathrm{b}$. When the absolute value of $\mathrm{r}$ is higher than 0.5 and $\mathrm{p}$ is lower than 0.05 , the correlations show strong correlation.

The results are as follows:

at $\mathrm{B}$ band, $\alpha=(0.80 \pm 0.03) \log F_{B}+(0.92 \pm 0.01)$, with $\mathrm{r}=0.68, p=4.23 \times 10^{-13}$; 

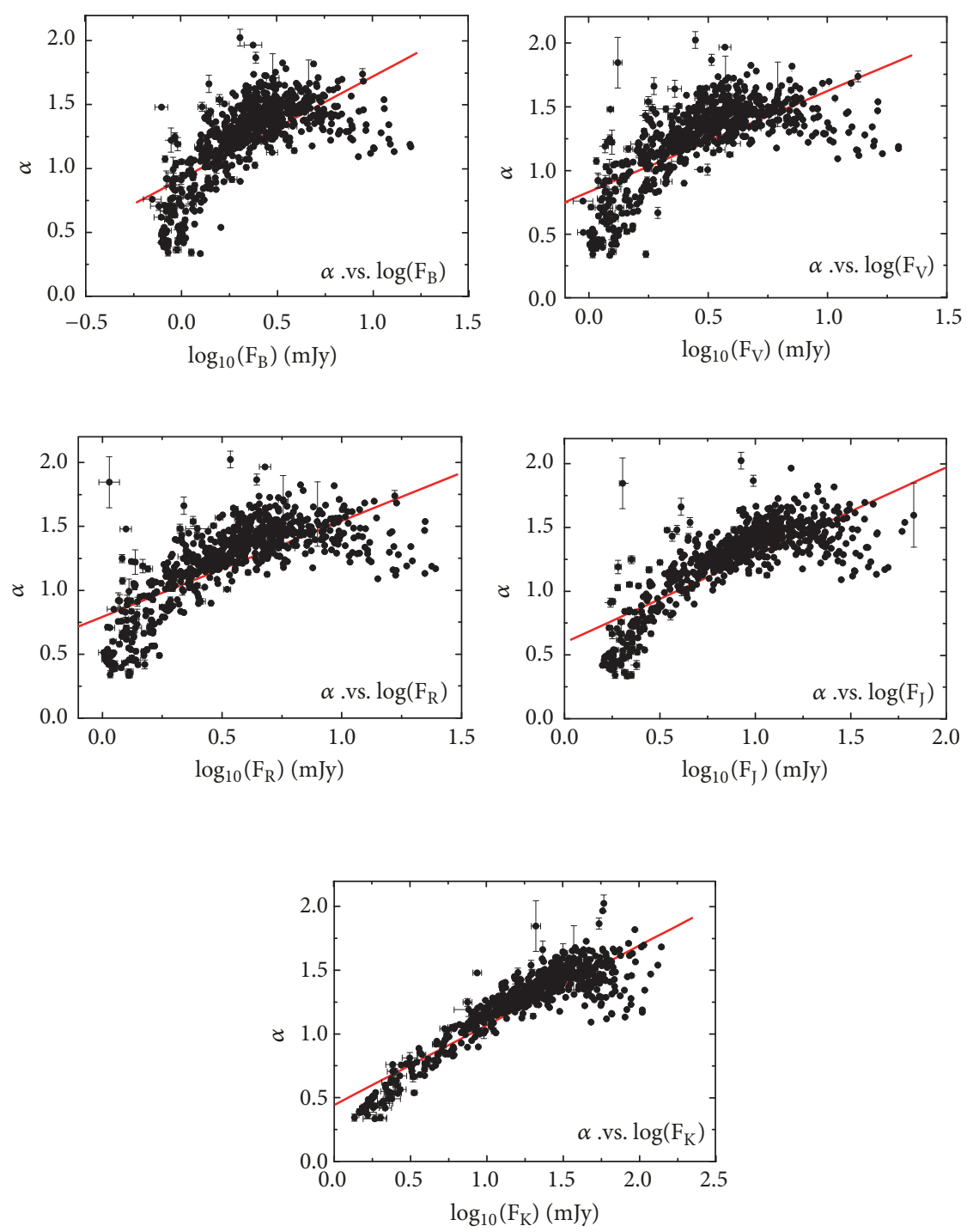

FIgURE 3: Relations between the logarithmic flux densities $\left(\log F_{v}\right)$ and optical spectral indices $(\alpha)$. The red lines stand for the linear fitting.

at $\mathrm{V}$ band, $\alpha=(0.79 \pm 0.03) \log F_{V}+(0.83 \pm 0.02)$, with $\mathrm{r}=0.70, p=1.19 \times 10^{-18}$;

at R band, $\alpha=(0.75 \pm 0.03) \log F_{R}+(0.78 \pm 0.02)$, with $\mathrm{r}=0.72, p=9.95 \times 10^{-22}$;

at J band, $\alpha=(0.69 \pm 0.02) \log F_{J}+(0.60 \pm 0.02)$, with $\mathrm{r}=0.75, p=6.08 \times 10^{-26}$;

at $\mathrm{K}$ band, $\alpha=(0.63 \pm 0.01) \log F_{K}+(0.44 \pm 0.01)$, with $\mathrm{r}=0.91, p=7.24 \times 10^{-34}$.

The fitting results are shown in Figure 3, with the red lines standing for the fitting lines.

\section{Discussion}

4.1. Optical Variability. The optically variable timescale is an important physical quantity and is often used to probe the physics process of blazars. Based on the optical-infrared lightcurves, we obtain the spectral indices $(\alpha)$ and analyze the long-term $\alpha$ variability; see Figure 4 (the upper subpicture). The spectral variability shows quasiperiodic properties, $P_{\alpha \mid 1}=0.55 \pm 0.08$ years, $P_{\alpha \mid 2}=0.85 \pm 0.07$ years, and $P_{\alpha \mid 3}=$ $1.21 \pm 0.06$ years, among which $P_{\alpha \mid 3}$ shows the strongest signal and is consistent with the result calculated from the lightcurves; see Figure 4 (the lower subpicture). The upper results show that the lightcurves and spectral variability have the same long-term variation tendencies.

If the long-term periodicity $(\boldsymbol{P})$ is caused by a slim disk, the periodicity can be expressed as $P / y r=$ $9.0 \delta(\alpha / 0.1)^{-0.62}\left(M_{B H} / 10^{6} M_{\odot}\right)^{1.37}$, where $\alpha$ is the viscosity coefficient [18], $P$ is periodicity (in unit of years), and $M_{B H}$ is the central black hole mass. The mass calculated from this method is about $\sim 10^{6} M_{\odot}$, which is smaller than the others, 

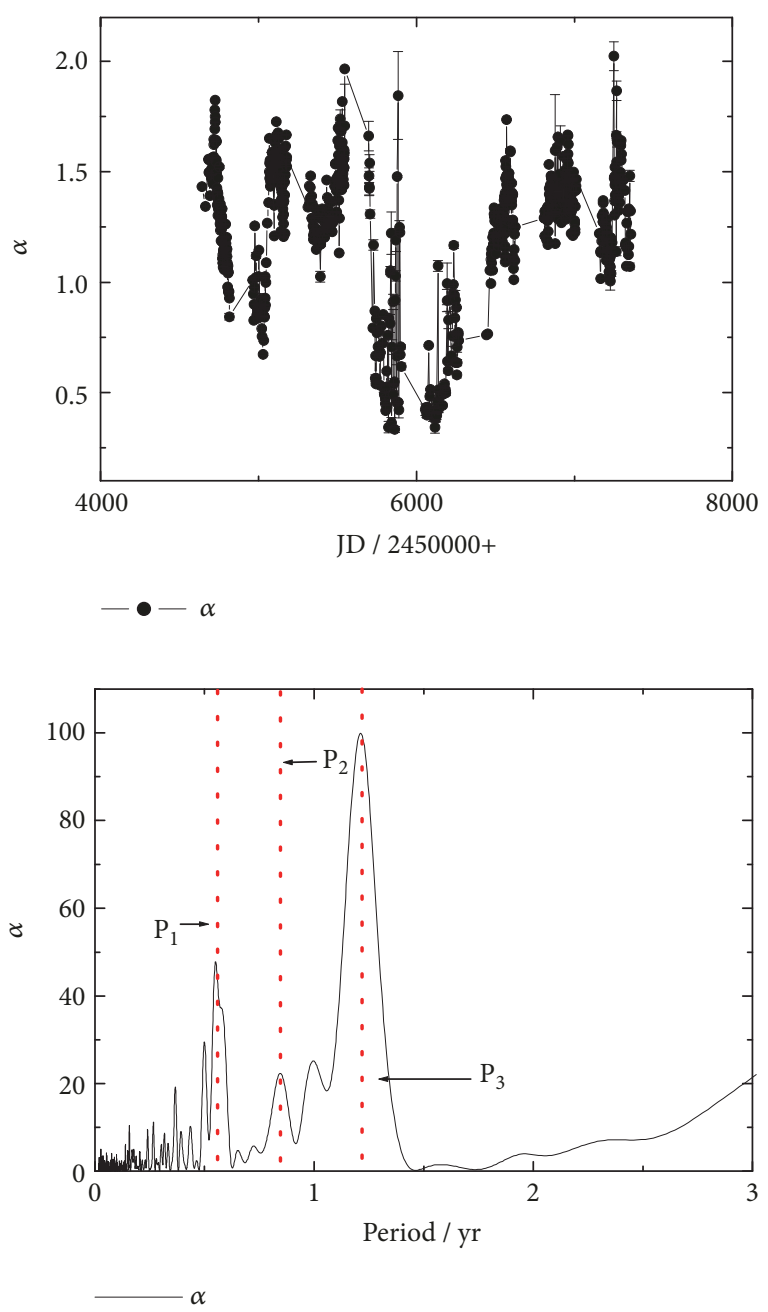

FIgURE 4: The $\alpha$ variability and period of 3C454.3. The upper subpicture stands for $\alpha$ variability and the lower subpicture stands for the quasiperiodicity, which are noted by the dot lines.

such as $1.49 \times 10^{9} M_{\odot}$ calculated by Woo et al. (2002) [30] and $5.01 \times 10^{9} M_{\odot}$ calculated by Sbarrato et al. (2012) [37].

Long-term periodicity might come from the influence of binary black hole. OJ287 is considered as a binary black system [38]. Some authors [39, 40] consider PKS 1510-089 as a binary black hole.

3C454.3 might be a binary black hole system. Based on the variability timescales, $\mathrm{Li}$ et al. (2007) [40] gave a method about how to calculate the masses of the primary black hole $\left(M_{1}, \mathrm{PBH}\right)$ and secondary black hole $\left(M_{2}, \mathrm{SBH}\right)$. The methods are as follows.

(1) The distance (a) between the two black holes can be calculated from the following relation:

$$
a=3 \times 10^{5} \times \frac{k M_{2} \times P}{2 \pi \times \Delta T \times M_{\odot}}(\mathrm{cm})
$$

where $\Delta T$ is variable timescale, $P$ is the quasiperiodicity, and $k$ is the ratio between the jet diameter and radius of secondary black hole.

(2) The radio (q) between the mass from the primary black hole and the one from the secondary black hole is

$$
q=\frac{M_{1}}{M_{2}}
$$

(3) For the binary black hole system, the following relation can be deduced

$$
M_{2}(1+q)=\frac{4 \pi^{2} a^{3}}{G P^{2}} .
$$

$\Delta T=0.685$ days is collected from [41]. Because the quasiperiodicity 1.24 years $(P=1.24)$ are shown both in the lightcurves and in the spectral variability, so we choose the periodicity being 1.24 years to calculate the binary black hole masses. Based on the expression (3), (4), (5), we can obtain

$$
M_{2}(1+q)=\frac{k^{3} M_{2}^{3}}{3.11 \times 10^{17} \times M_{\odot}^{2}} .
$$

So, the masses should be

$$
\begin{aligned}
& M_{1}=5.57 q \times 10^{8} \sqrt{\frac{1+q}{k^{3}}} M_{\odot}, \\
& M_{2}=5.57 \times 10^{8} \sqrt{\frac{1+q}{k^{3}}} M_{\odot} .
\end{aligned}
$$

If the total mass is $M=1.49 \times 10^{9} M_{\odot}$ [30], we can obtain $M=M_{1}+M_{2}=5.57 \times 10^{8} \sqrt{\left((1+q)^{3} / k^{3}\right)} M_{\odot}=1.49 \times 10^{9} M_{\odot}$. When Sillanpaa et al. (1988) [38] analyzed the binary black hole system of OJ287, $\mathrm{q}=200$ was used. If we take $\mathrm{q}=200$, the binary black holes masses are $M_{1}=1.48 \times 10^{9} M_{\odot}, M_{2}=$ $7.45 \times 10^{6} M_{\odot}$.

4.2. Time Delay among Different Bands. The analysis about the multiband time delays can help us to study the emission properties and can reflect the terms of electron cooling timescales [42, 43]. For S5 0716+714, Gupta et al. (2012) [44] calculated the time delays between $\mathrm{V}$ and $\mathrm{R}$ bands and obtained $\tau_{V R}=1.4$ days and -1.4 days. For $S 50716+714, \mathrm{Wu}$ et al. (2012) [45] obtained the time delays, $\tau_{B V}=-9.76 \sim 5.48$ minutes and $\tau_{V R}=0.77 \sim 25.35$ minutes. For 1ES 1959+650, Yuan et al. (2015) [9] obtained $\tau_{R I}=4.73 \pm 2.75$ days, $\tau_{V R}=$ $1.38 \pm 2.92$ days, and $\tau_{V I}=4.97 \pm 3.87$ days.

We avail the DCF method $[46,47]$ to explore time delays among different bands. In order to get the delay time more accurately, we use three methods to fit the DCF results: local polynomial regression (LOESS) [48], local Kernel regression (LOCFIT) [49], and Gaussian regression. The fitting curves have been added in Figure 5, in which red lines stand for Gaussian regression, blue lines stand for Locfit regression, 

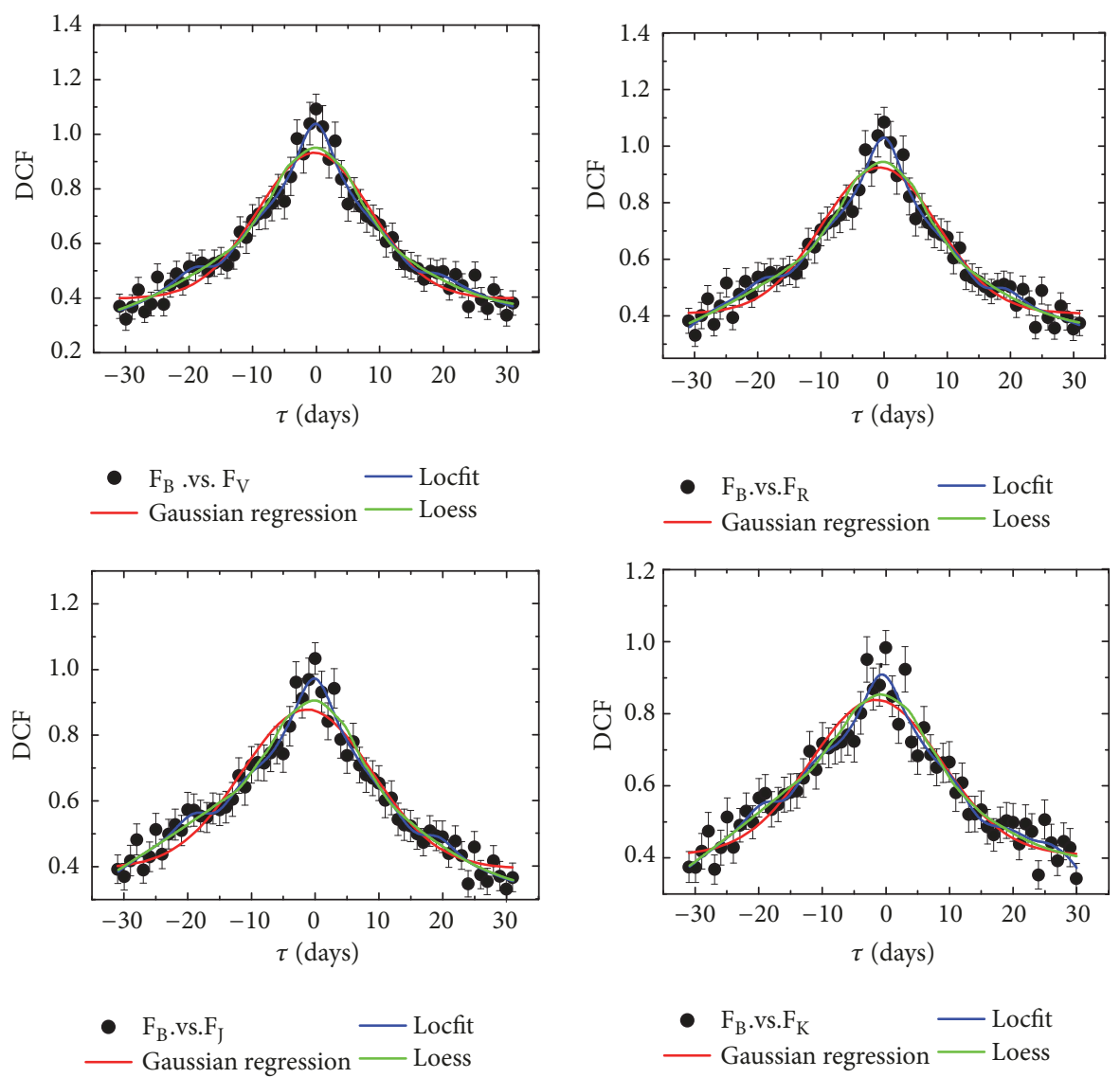

Figure 5: The time delays among the different bands. The red lines stand for the Gaussian fitting, the blue lines stand for Locfit regression, and green lines stand for Loess regression.

and green lines stand for Loess regression, based on which we can obtain $\tau_{V B}=0.15 \pm 0.12$ days, $\tau_{R B}=0.37 \pm 0.28$ days, $\tau_{J B}=0.58 \pm 0.40$ days, and $\tau_{K B}=1.02 \pm 0.47$ days. Our results show that the B-band lightcurves are ahead of the other bands.

4.3. Relation between Flux Density and Spectral Index. In this work, we analyze the relations between flux densities and spectral indices. At the whole five bands $(B, V, R, J, K)$, $\log F_{v}$ and $\alpha$ show strong correlations. When the source turn to be brighter, the spectrum turn to be redder, which is a typical RWB behavior and consistent with the other FSRQs $[32,50,51]$.

We check those distributions and find that there lie break points; see Figure 6 . We use a broken power law to fit them and obtain the break points: $\log _{10}\left(F_{b \mid B}\right)=0.58$ $\mathrm{mJy}, \log _{10}\left(F_{b \mid V}\right)=0.69 \mathrm{mJy}, \log _{10}\left(F_{b \mid R}\right)=0.78 \mathrm{mJy}$, $\log _{10}\left(F_{b \mid J}\right)=1.12 \mathrm{mJy}, \log _{10}\left(F_{b \mid K}\right)=1.49 \mathrm{mJy}$, which are noted in Figure 6 . When the source becomes fainter, the relations show correlations and when the source becomes brighter, their relations turn to be anticorrelations.

In this paper, we use the observations of 3C454.3 from SMARTs to analyze the lightcurves and calculate the optical spectral indices. Our results show that except $\mathrm{K}$ band, the largest variances at $\mathrm{B}, \mathrm{V}, \mathrm{R}$, and $\mathrm{J}$ bands are similar (about 3.5 mag) within about 3 years. The long-term bright variability and spectral variability have the same quasiperiodicities. There lie time delays among different optical and near-IR bands (B, V, R, J, K).

\section{Data Availability}

The text formatting data used to support the findings of this study have been deposited in the "http://www.astro.yale.edu/ smarts/glast/home.php" repository. The data on the upper website are public and may be used by other investigators. If others intend to make use of the data, please inform the SMARTs group by email to glast@elilists.yale.edu and send copies of any resulting publications, including telegrams. Please acknowledge them in your paper by including a citation to the Bonning et al. (2012), mentioned above, and with the following: "This paper has made use of upto-date SMARTS optical/near-infrared lightcurves that are available at http://www.astro.yale.edu/smarts/glast/home .php." Please also include "SMARTS" as a facility keyword. 

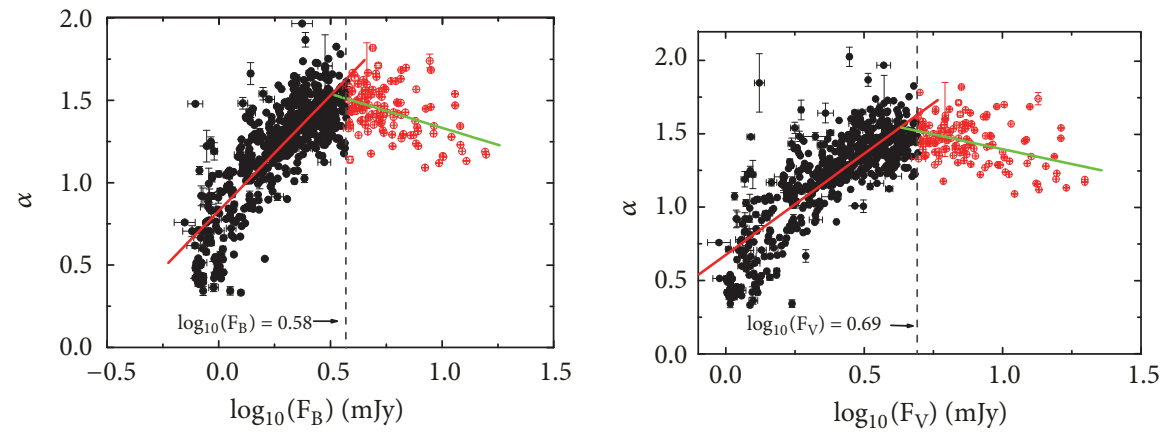

$$
\begin{aligned}
& -\log _{10}\left(F_{B}\right)<0.58 \\
& \circ \log _{10}\left(F_{B}\right)>0.58
\end{aligned}
$$

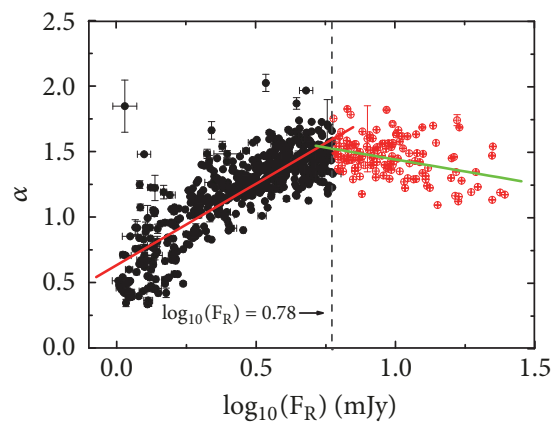

- $\log _{10}\left(\mathrm{~F}_{\mathrm{R}}\right)<0.78$

$\circ \log _{10}\left(\mathrm{~F}_{\mathrm{R}}\right)>0.78$

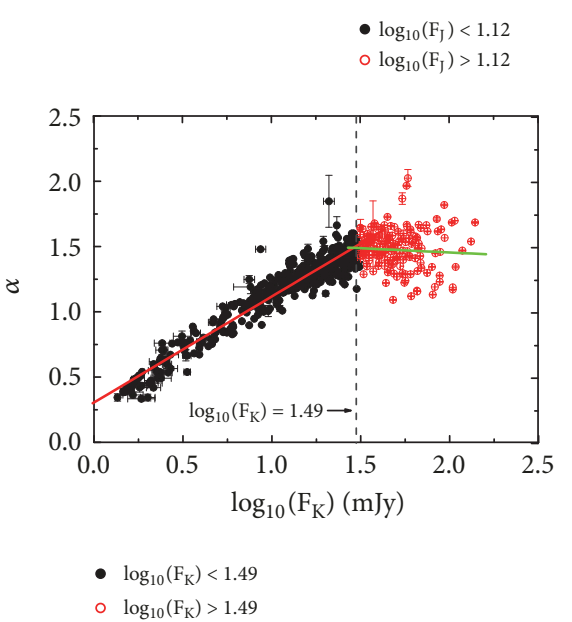

FiguRE 6: Relations between the logarithmic flux densities $\left(\log \left(F_{\gamma}\right)\right)$ and optical spectral indices $(\alpha)$. The black dots and red dots stand for the different regions, with the blue line separating them.

\section{Conflicts of Interest}

The authors declare that they have no conflicts of interest.

\section{Acknowledgments}

The work is partially supported by the National Natural Science Foundation of China (NSFC 11403006, NSFC 11733001, NSFC U1831119, NSFC U1531245 and NSFC 11503004), Science and Technology Program of Guangzhou (201707010401), Innovative Training Program of Guangzhou
University, Innovation Team in Guangdong Province (2014KCXD014), and the financial support for the key subject of Guangzhou City. This paper has made use of up-to-date SMARTS optical/near-infrared lightcurves that are available at http://www.astro.yale.edu/smarts/glast/home.php.

\section{References}

[1] C. M. Urry and P. Padovani, "Unified schemes for radio-loud active galactic nuclei," Publications of the Astronomical Society of the Pacific, vol. 107, pp. 803-882, 1995. 
[2] M. H. Ulrich, L. Maraschi, and C. M. Urry, "Variability of active galactic nuclei," Annual Review of Astronomy and Astrophysics, vol. 35, pp. 445-502, 1997.

[3] M. Stickel, J. W. Fried, H. Kuehr, P. Padovani, and C. M. Urry, "The complete sample of 1 Jansky BL Lacertae objects. I Summary properties," The Astrophysical Journal, vol. 374, p. 431, 1991.

[4] R. A. Edelson, J. H. Krolik, and G. F. Pike, "Broad-band properties of the CfA Seyfert galaxies. III. Ultraviolet variability," The Astrophysical Journal, vol. 359, no. 1, pp. 86-97, 1990.

[5] D. Trevese and F. Vagnetti, "Quasar spectral slope variability in the optical band," The Astrophysical Journal, vol. 564, pp. 624630, 2002.

[6] F. Vagnetti, D. Trevese, and R. Nesci, "Spectral slope variability of BL Lacertae objects in the optical band," The Astrophysical Journal, vol. 590, no. 1 I, pp. 123-127, 2003.

[7] B. Z. Dai, X. H. Li, Z. M. Liu et al., "The long-term multiband optical observations and colour index for the quasar 3C 273," Monthly Notices of the Royal Astronomical Society, vol. 392, no. 3, pp. 1181-1192, 2009.

[8] K. Poon, J. H. Fan, and J. N. Fu, "The optical microvariability and spectral changes of the BL Lacertae object S5 0716+714," The Astrophysical Journal Supplement Series, vol. 185, pp. 511525, 2009.

[9] Y. H. Yuan, J. H. Fan, and H. J. Pan, "Optical photometry of the BL Lac object 1ES 1959+650," The Astronomical Journal, vol. 150, no. 3, p. 67, 2015.

[10] Y. H. Yuan, J. H. Fan, H. J. Pan, Z. Y. Peng, Y. Liu, and H. B. Xiao, "Optical monitoring and spectral analysis of the Seyfert galaxy NGC1275," Astrophysics and Space Science, vol. 360, p. 9, 2015.

[11] J. G. Kirk and A. Mastichiadis, "Variability patterns of synchrotron and inverse Compton emission in blazars," Astroparticle Physics, vol. 11, no. 1-2, pp. 45-48, 1999.

[12] A. Mastichiadis and J. G. Kirk, "Variability in the synchrotron self-Compton model of blazar emission," Astronomy \& Astrophysics, vol. 320, pp. 19-25, 1997.

[13] J. G. Kirk, F. M. Rieger, and A. Mastichiadis, "Particle acceleration and synchrotron emission in blazar jets," Astronomy \& Astrophysics, vol. 333, no. 2, pp. 452-458, 1998.

[14] W. Cui, "X-Ray flaring activity of markarian 421," The Astrophysical Journal, vol. 605, no. 2, pp. 662-669, 2004.

[15] R. Sato, J. Kataoka, T. Takahashi, G. M. Madejski, S. Rügamer, and S. J. Wagner, The Astrophysical Journal, vol. 680, no. 1, pp. L9-L12, 2008.

[16] M. Fiorucci, S. Ciprini, and G. Tosti, "The continuum spectral features of blazars in the optical region," Astronomy \& Astrophysics, vol. 419, no. 1, pp. 25-34, 2004.

[17] J. H. Fan, O. Kurtanidze, Y. Liu, G. M. Richter, R. Chanishvili, and Y. H. Yuan, "Optical monitoring of two brightest nearby quasars, PHL 1811 and 3C 273," The Astrophysical Journal Supplement Series, vol. 213, no. 2, 2014.

[18] J. Fan, "Optical variability of blazars," Chinese Journal of Astronomy and Astrophysics, vol. 5, no. S1, pp. 213-223, 2005.

[19] N. Jackson and W. A. Browne, "Optical properties of quasars. I - Observations. II - Emission-line geometry and radio properties," Monthly Notices of the Royal Astronomical Society, vol. 250, pp. 414-421, 1991.

[20] S. G. Jorstad, A. P. Marscher, V. M. Larionov et al., "Flaring behavior of the quasar 3C 454.3 across the electromagnetic spectrum," The Astrophysical Journal, vol. 715, no. 1, pp. 362$384,2010$.
[21] Y. Zhou, D.-H. Yan, and B.-Z. Dai, “The optical variability properties of flat spectrum radio quasar 3C 454.3," New Astronomy, vol. 36, pp. 19-25, 2015.

[22] C. M. Raiteri, "WEBT and XMM-Newton observations of 3C 454.3 during the post-outburst phase. Detection of the little and big blue bumps," Astronomy \& Astrophysics, vol. 473, pp. 819827, 2007.

[23] C. M. Raiteri, "Radio-to-UV monitoring of AO $0235+164$ by the WEBT and Swift during the 2006-2007 outburst," Astronomy \& Astrophysics, vol. 480, pp. 339-347, 2008.

[24] E. W. Bonning, C. Bailyn, C. M. Urry et al., "Correlated variability in the blazar 3C 454.3," The Astrophysical Journal, vol. 697, no. 2, pp. L81-L85, 2009.

[25] C. M. Raiteri, M. Villata, M. F. Aller, and M. A. Gurwell, "The long-lasting activity of 3C 454.3. GASP-WEBT and satellite observations in 2008-2010," Astronomy \& Astrophysics, vol. 534, p. 87, 2011.

[26] J. R. Webb, A. G. Smith, R. J. Leacock, G. L. Fitzgibbons, P. P. Gombola, and D. W. Shepherd, "Optical observations of 22 violently variable extragalactic sources - 1968-1986," The Astronomical Journal, vol. 95, p. 374, 1988.

[27] A. Ciaramella, "A multifrequency analysis of radio variability of blazars," Astronomy \& Astrophysics, vol. 419, pp. 485-500, 2004.

[28] J. H. Fan, Y. Liu, and Y. H. Yuan, "Radio variability properties for radio sources," Astronomy \& Astrophysics, vol. 462, pp. 547-552, 2007.

[29] N. A. Kudryavtseva and T. B. Pyatunina, "A search for periodicity in the light curves of selected blazars," Astronomy Reports, vol. 50, pp. 1-11, 2006.

[30] J. H. Woo and C. M. Urry, "Active galactic nucleus black hole masses and bolometric luminosities," The Astrophysical Journal, vol. 579, p. 530, 2002.

[31] L. M. Brown, E. I. Robson, W. K. Gear, and M. G. Smith, "Multifrequency observations of blazars. IV - The variability of the radio to ultraviolet continuum," The Astrophysical Journal, vol. 340, p. 150, 1989.

[32] E. Bonning, C. M. Urry, C. Bailyn et al., "Smarts optical and infrared monitoring of 12 gamma-ray bright blazars," The Astrophysical Journal, vol. 756, no. 1, p. 13, 2012.

[33] T. J. Deeming, "Fourier analysis with unequally-spaced data," Astrophysics and Space Science, vol. 36, pp. 137-156, 1975.

[34] N. R. Lomb, "Least-squares frequency analysis of unequally spaced data," Astrophysics and Space Science, vol. 39, no. 2, pp. 447-462, 1976.

[35] J. D. Scargle, "Studies in astronomical time series analysis. II-statistical aspects of spectral analysis of unevenly spaced data," The Astrophysical Journal, vol. 263, pp. 835-853, 1982.

[36] M. Schulz and M. Mudelsee, "REDFIT: Estimating red-noise spectra directly from unevenly spaced paleoclimatic time series," Computers \& Geosciences, vol. 28, no. 3, pp. 421-426, 2002.

[37] T. Sbarrato, G. Ghisellini, L. Maraschi, and M. Colpi, "The relation between broad lines and $\gamma$-ray luminosities in Fermi blazars," Monthly Notices of the Royal Astronomical Society, vol. 421, no. 2, pp. 1764-1778, 2012.

[38] A. Sillanpaa, S. Haarala, M. J. Valtonen, B. Sundelius, and G. G. Byrd, "OJ 287 - Binary pair of supermassive black holes," The Astrophysical Journal, vol. 325, p. 628, 1988.

[39] M. F. Gu, X. Cao, and D. R. Jiang, "Relation between blue/ ultraviolet continuum shape and the ratio of radio-to-optical emission for B3-VLA quasars," Monthly Notices of the Royal Astronomical Society, vol. 321, p. 369, 2001. 
[40] J. Li, J. H. Fan, and Y. H. Yuan, "The parameters of binary black hole systemin PKS 1510-089,” ChPhy, vol. 16, p. 877, 2007.

[41] X. Fan, S. Li, N. Liao et al., "Optical and Gamma-Ray Variability Behaviors of 3C 454.3 from 2006 to 2011," The Astrophysical Journal, vol. 856, no. 1, p. 80, 2018.

[42] J. M. Bai and M. G. Lee, "Radio/X-ray offsets of large-scale jets caused by synchrotron time lags," The Astrophysical Journal, vol. 585, pp. 113-116, 2003.

[43] B. K. Zhang, S. Wang, X. Y. Zhao, B. Z. Dai, and M. Zha, "Longterm optical and infrared variability of the BL Lac object PKS 0537 - 441," Monthly Notices of the Royal Astronomical Society, vol. 428, pp. 3630-3635, 2013.

[44] A. C. Gupta, T. P. Krichbaum, and P. J. Wiita, "Multiwavelength intraday variability of the BL Lacertae S5 0716+714," Monthly Notices of the Royal Astronomical Society, vol. 425, pp. 1357-1370, 2012.

[45] J. Wu, M. Böttcher, X. Zhou, X. He, J. Ma, and Z. Jiang, "Simultaneous $\mathrm{B} / \mathrm{V} / \mathrm{R} /$ monitoring of bl lacertae object S5 $0716+714$ and detection of inter-band time delay," The Astronomical Journal, vol. 143, no. 5, 2012.

[46] R. A. Edelson and J. H. Krolik, "The discrete correlation function - A new method for analyzing unevenly sampled variability data," The Astrophysical Journal, vol. 333, pp. 646-659, 1988.

[47] B. R. Hufnagel and J. N. Bregman, "Optical and radio variability in blazars," The Astrophysical Journal, vol. 386, pp. 473-484, 1992.

[48] W. S. Cleveland, Visualizing Data, Hobart Press, 1993.

[49] C. Loader, Local regression and likelihood, Statistics and Computing, Springer-Verlag, New York, 1999.

[50] A. Ramírez, J. A. De Diego, D. Dultzin-Hacyan, and J. N. González-Pérez, “Optical variability of PKS 0736+017," Astronomy \& Astrophysics, vol. 421, no. 1, pp. 83-89, 2004.

[51] M. F. Gu, C.-U. Lee, S. Pak et al., "Multi-colour optical monitoring of eight red blazars," Astronomy \& Astrophysics, vol. 450, pp. 39-51, 2006. 

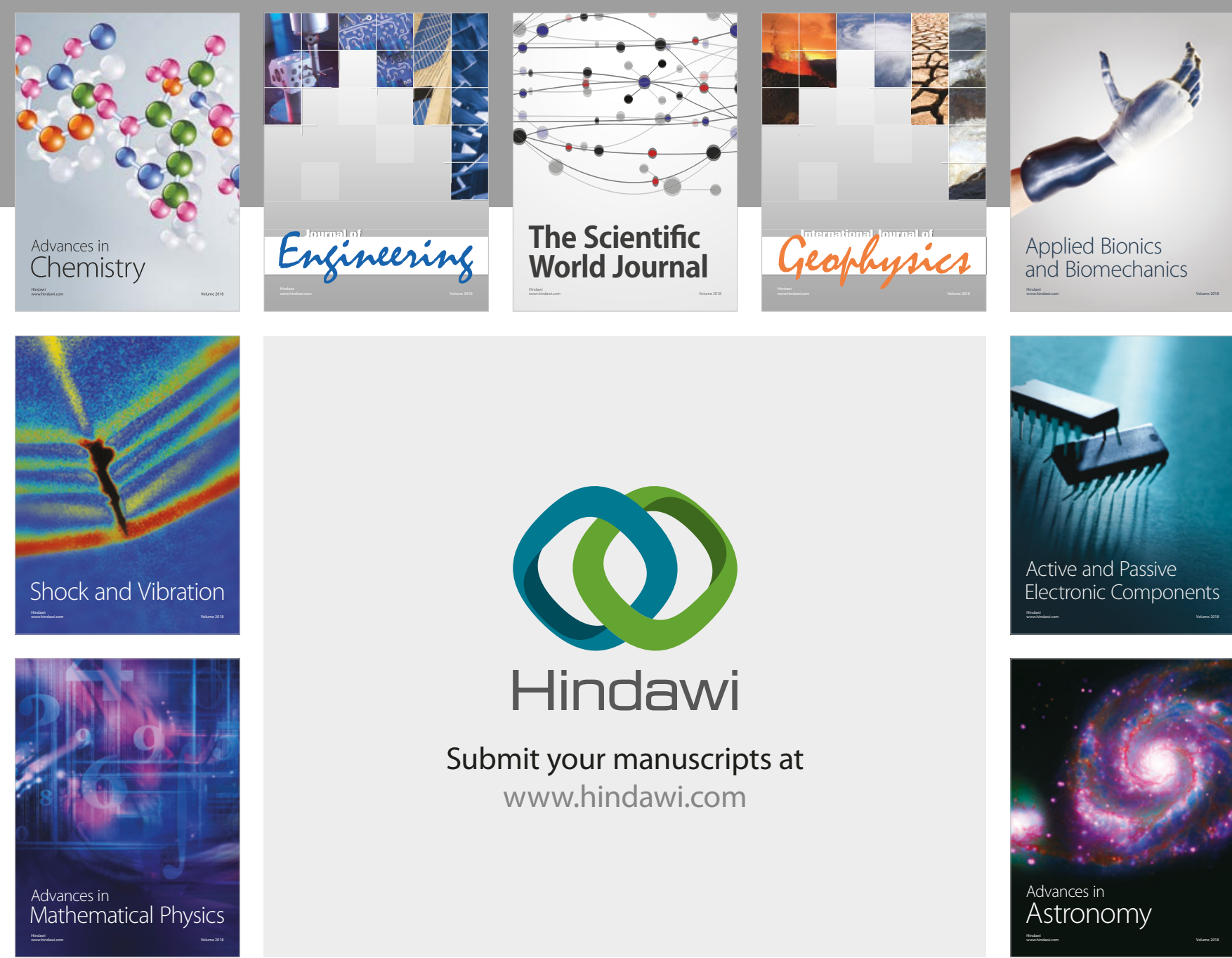

Submit your manuscripts at

www.hindawi.com

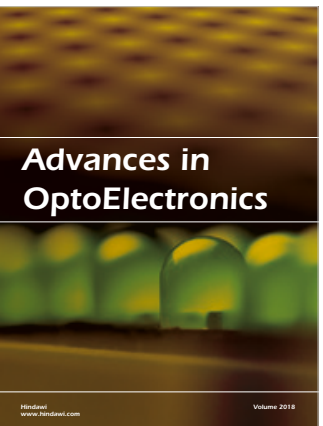

\section{Rotcting Machinery}
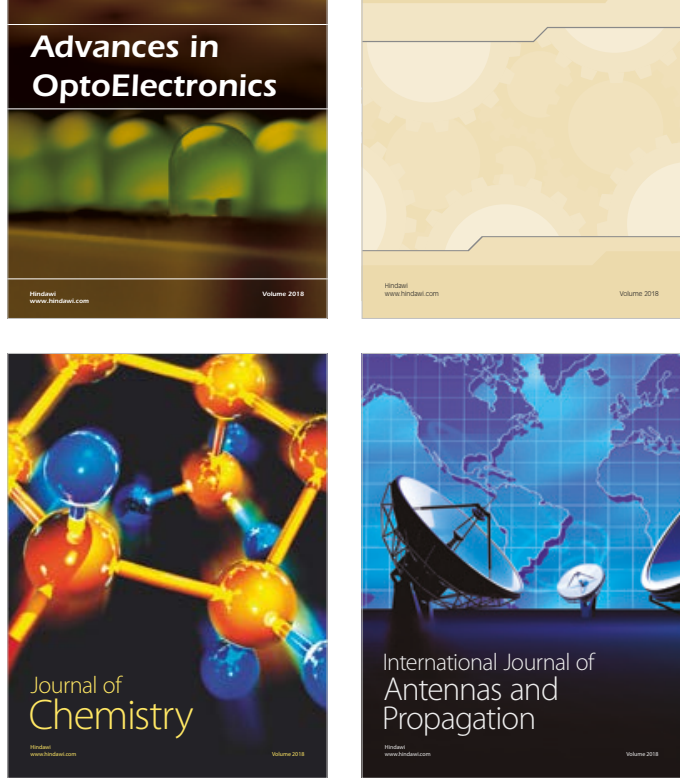

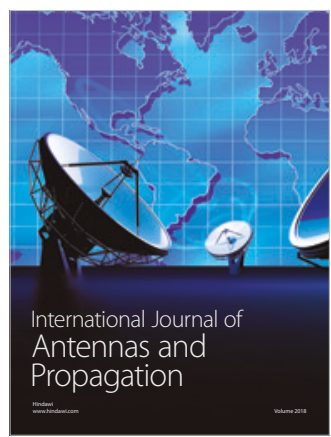

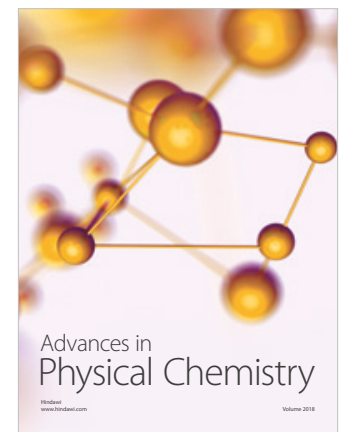

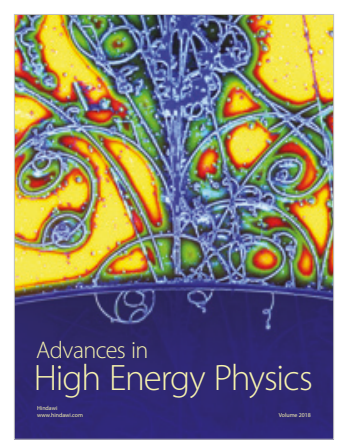

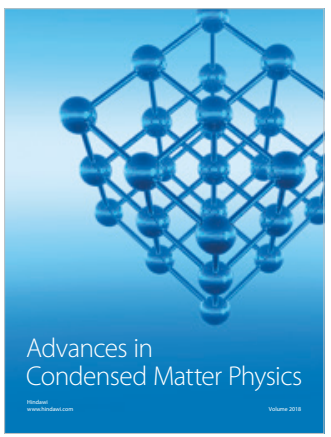

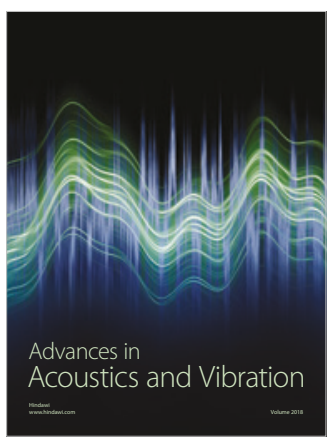

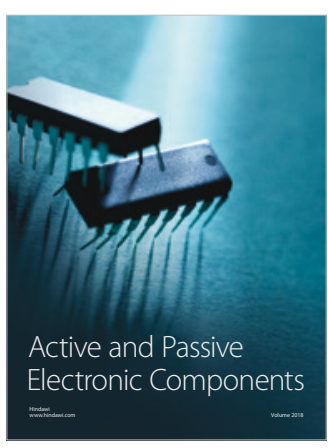
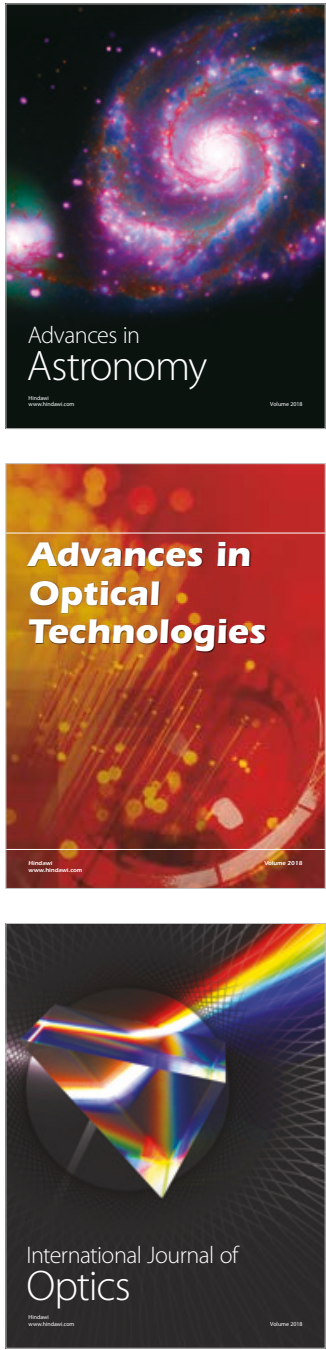